\title{
Soft Pneumatic Gelatin Actuator for Edible Robotics
}

\author{
Jun Shintake ${ }^{1}$, Harshal Sonar ${ }^{2}$, Egor Piskarev ${ }^{3}$, Jamie Paik $^{2}$ and Dario Floreano ${ }^{1}$
}

\begin{abstract}
We present a fully edible pneumatic actuator based on gelatin-glycerol material. The actuator is monolithic, fabricated via a molding process, and measures $90 \mathrm{~mm}$ in length, $20 \mathrm{~mm}$ in width, and $17 \mathrm{~mm}$ in thickness. Thanks to the material mechanical characteristics similar to those of silicone elastomers, the actuator exhibits a bending angle of $170.3^{\circ}$ and a blocked force of $0.34 \mathrm{~N}$ at the applied pressure of $25 \mathrm{kPa}$. These values are comparable to elastomer based pneumatic actuators. As a validation example, two actuators are integrated to form a gripper capable of handling various objects, highlighting the high performance and applicability of the edible actuator. These edible actuators, combined with other recent edible materials and electronics, could lay the foundation for a new type of edible robots.
\end{abstract}

\section{INTRODUCTION}

Revisiting the material foundations of robotic components can lead to novel functionalities and application fields. For example, soft robotics, where robots are composed of compliant materials has led to superior features, such as improved mechanical robustness and simplified structure and control, and safer and more compliant functionalities [1], [2].

Here we propose the use of edible materials for a new type of robotic architectures, which we call "Edible Robotics". Edible robots can be biodegradable, biocompatible, and environmentally sustainable with none or lower level of toxicity. These functionalities can already be seen in organic electronics made of edible materials [3] to fabricate transistors [4], sensors [5], batteries [6]-[9], electrodes [10], and capacitors [11]. The (still missing) availability of edible actuators could pave the way to fully edible robots. The components of such edible robots could also me mixed with nutrient or pharmaceutical components for digestion and metabolization. Potential applications are disposable robots for exploration (as also mentioned in [12], [13]), digestible robots for medical purposes in humans and animals, and food transportation where the robot does not require additional payload because the robot is the food.

So far, the use of edible materials in robotics remains very limited. Researchers have developed a gelatin hydrogel actuator immersed in $\mathrm{NaOH}$ solution [12], and an ingestible robot that uses intestines of pigs as folding parts [14]. Both

This work was supported by the Swiss National Centre of Competence in Research (NCCR) Robotics.

${ }^{1}$ J. Shintake and D. Floreano are with the Laboratory of Intelligent Systems (LIS), École Polytechnique Fédérale de Lausanne, Route Cantonale, Lausanne 1015, Switzerland. jun. shintakedepfl.ch

${ }^{2}$ H. Sonar and J. Paik are with the Reconfigurable Robotics Laboratory (RRL), École Polytechnique Fédérale de Lausanne, Route Cantonale, Lausanne 1015, Switzerland.

${ }^{3}$ E. Piskarev is with the Section of Mechanical Engineering, École Polytechnique Fédérale de Lausanne, Route Cantonale, Lausanne 1015, Switzerland.

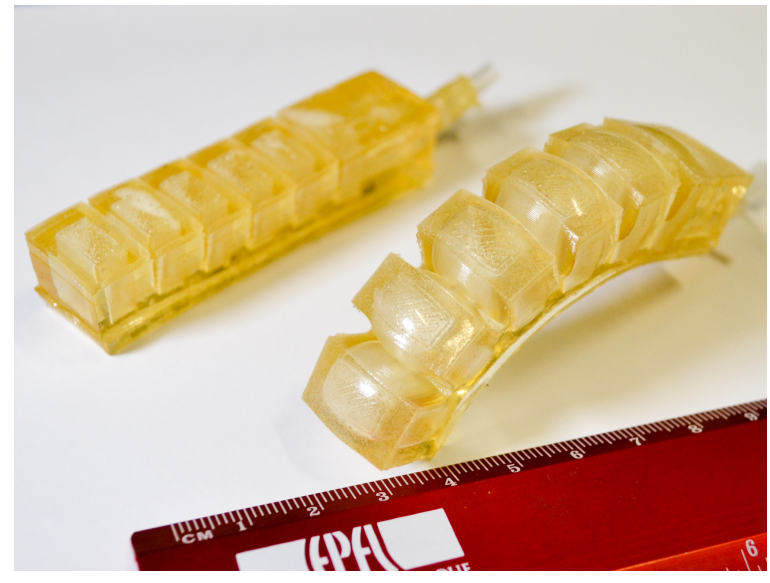

Fig. 1. Edible soft pneumatic actuator developed in this study. (left) actuator in the non-pressurized state. (right) actuator in a pressurized state.

cases require external electric and magnetic fields, which can make them challenging to use outside of a specific environment.

In this paper, we describe a soft gelatin pneumatic actuator for edible robotics. Soft actuators, especially the ones with pneumatic configuration, have being widely developed and applied in various robotic applications thanks to their simple structure that can be extended to form a robot itself [1], [2]. Therefore, developing edible soft pneumatic actuator is a promising approach that can immediately lead to realization of edible robots. Gelatin is an edible material, and can be polymerized when an edible plasticizer, (glycerol in this work), is used to form a protein network [15]. Gelatinglycerol material could replace silicone elastomers, a compliant material often used in soft robots. Once dissolved into aqueous solution, the material can be used in molding fabrication of soft actuators in the same manner as more traditional elastomers.

In Section II, we perform tensile testing of the gelatinglycerol material in order to investigate if its major mechanical properties such as, the Young's modulus, tensile strength, and elongation at break, are comparable to that of silicone elastomers. These results are important for the applicability of the proposed edible material composition for real world robotic applications. In Section III, we develop a proof-ofconcept fully edible soft pneumatic actuator, and characterize its performance in terms of the bending angle and the blocked force as functions of the applied pressure. In Section IV, we show the integration of two edible actuators to form a two-finger gripper capable of grasping various objects. We discuss the results in Section $\mathrm{V}$ and draw conclusions in Section VI. 


\section{Characterization OF GELATIN-GLYCEROL MEMBRANE}

In this section, the mechanical properties of the gelatinglycerol material is investigated by performing tensile testing.

\section{A. Sample preparation}

Gelatin (48723) and glycerol (G5516) were purchased from Sigma Aldrich (Switzerland). These materials were used as received. Aqueous solutions containing gelatin (GEL), glycerol (GLY), and distilled water (WAT) were prepared by mixing the contents for $30 \mathrm{~min}$ at $80{ }^{\circ} \mathrm{C}$. Two different solutions were prepared: GEL/GLY/WAT $=1: 1: 8$ and 1:2:8. The solutions were cast on an acrylic mold $(20 \mathrm{~g}$ solution/mold) and dried at room temperature $\left(25{ }^{\circ} \mathrm{C}\right)$ for $48 \mathrm{~h}$ inside a chemical hood. After drying, a membrane thickness of $0.5 \mathrm{~mm}$ was formed in the mold. The membrane was then cut by a laser machine (Speedy 300, Trotec Laser) to obtain the dogbone shaped samples (Fig. 2) for uniaxial tensile testing. This dogbone shape sample was fabricated according to ASTM guidelines [16], and had reference marks for optical extensometer. The drying time of $48 \mathrm{~h}$ was experimentally determined prior to the sample preparation, based on the changes observed for mass of the membrane, which became almost constant at $48 \mathrm{~h}$ (Fig. 3(a)).

\section{B. Tensile testing}

Tensile testing of the samples was performed using a mechanical testing machine (5965, Instron) with the aid of optical extensometer. 5 samples were measured for each composition (GEL/GLY = 1:1 and 1:2). Every sample was pulled uniaxially until it broke (elongation at break), while recording the elongation and the loading. In order to obtain Young's modulus and the tensile strength of the gelatinglycerol material, the Yeoh hyperelastic material model [17] was fit to the measured data.

The strain energy density function of the Yeoh model is written as

$$
W=\sum_{i=1}^{3} C_{i}\left(I_{1}-3\right)^{i}
$$

where, $C_{i}$ are material constants. $I_{1}$ is the strain invariant

$$
I_{1}=\lambda_{1}^{2}+\lambda_{2}^{2}+\lambda_{3}^{2},
$$

where, $\lambda_{1}, \lambda_{2}$, and $\lambda_{3}$ are the stretch ratio in the length (tensile direction), the width, and the thickness direction, respectively, as represented in Fig. 2. The stretch ratios are defined as

$$
\lambda_{1}=\frac{l}{l_{0}}, \lambda_{2}=\frac{w}{w_{0}}, \lambda_{3}=\frac{h}{h_{0}},
$$

where, $l$ is the length, $w$ the width, and $h$ is the thickness of the sample. The subscription 0 stands for their initial state.

We assume the gelatin-glycerol material to be incompressible, that is

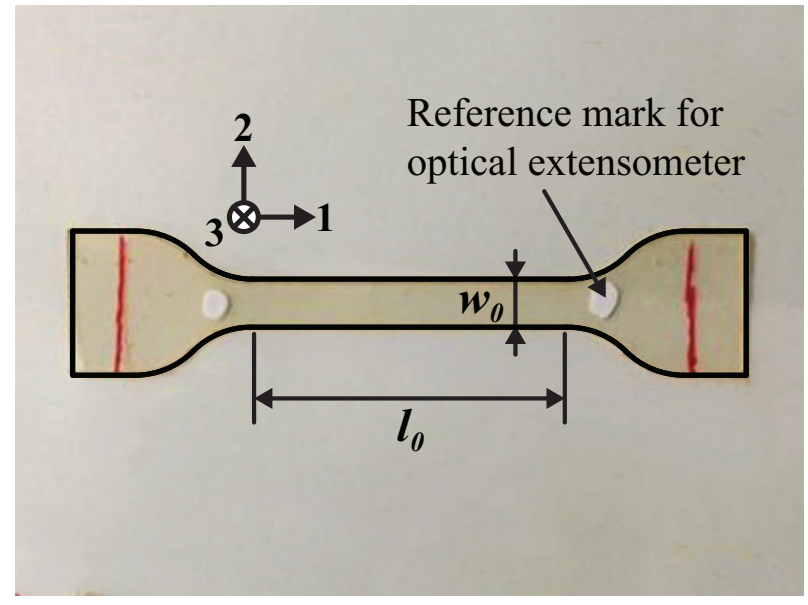

Fig. 2. A sample of gelatin-glycerol material for the tensile testing. Each sample has the same dimensions: length $l_{0}=35 \mathrm{~mm}$, width $w_{0}=5 \mathrm{~mm}$, and thickness $h_{0}=0.5 \mathrm{~mm}$.

$$
\lambda_{1} \lambda_{2} \lambda_{3}=1
$$

Since the sample is pulled uniaxially, the strains in the width and the thickness directions are the same

$$
\lambda_{2}=\lambda_{3}=\frac{1}{\sqrt{\lambda_{1}}} .
$$

By substituting Eq. 5 and Eq. 2 into Eq. 1, we get an expression of the strain energy density as a function of $\lambda_{1}$

$$
W=\sum_{i=1}^{3} C_{i}\left(\lambda_{1}^{2}+\frac{2}{\lambda_{1}}-3\right)^{i} .
$$

With the consideration of the Cauchy stress for incompressible Yeoh hyperelastic material model and uniaxial stretch, the stress in the pulling direction $\sigma_{1}$ is given by

$\sigma_{1}=\lambda_{1} \frac{\partial W}{\partial \lambda_{1}}=2\left(\lambda_{1}^{2}-\frac{1}{\lambda_{1}}\right) \sum_{i=1}^{3} i C_{i}\left(\lambda_{1}^{2}+\frac{2}{\lambda_{1}}-3\right)^{i-1}$.

The uniaxial loading $F_{1}$ is then expressed as

$$
F_{1}=2 h_{0} w_{0}\left(\lambda_{1}-\frac{1}{\lambda_{1}^{2}}\right) \sum_{i=1}^{3} i C_{i}\left(\lambda_{1}^{2}+\frac{2}{\lambda_{1}}-3\right)^{i-1} .
$$

Once the loading is measured, the tensile strength $\sigma_{\mathrm{TS}}$ at elongation at break $\lambda_{\mathrm{EAB}}$ is given as

$$
\sigma_{\mathrm{TS}}=\frac{F_{1 \mathrm{~m}}\left(\lambda_{\mathrm{EAB}}\right)}{w_{0} h_{0}},
$$

where $F_{1 \mathrm{~m}}$ is the measured uniaxial loading at $\lambda_{\mathrm{EAB}}$. Fitting Eq. 8 to the measured loading data gives us the material constants $C_{1}, C_{2}$, and $C_{3}$. The material constants are then used to obtain Young's modulus based on the consistency condition for the Yeoh hyperelastic material model

$$
2 C_{1}=\mu
$$


$\mathbf{a}$

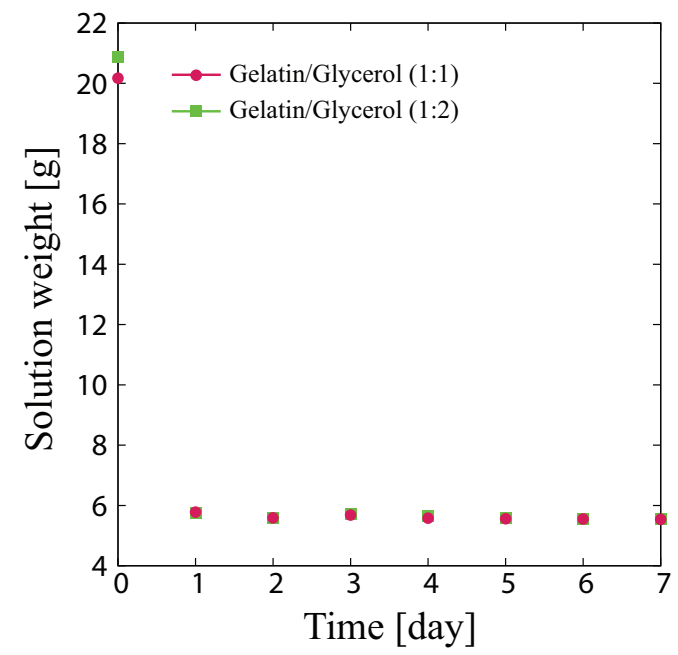

b

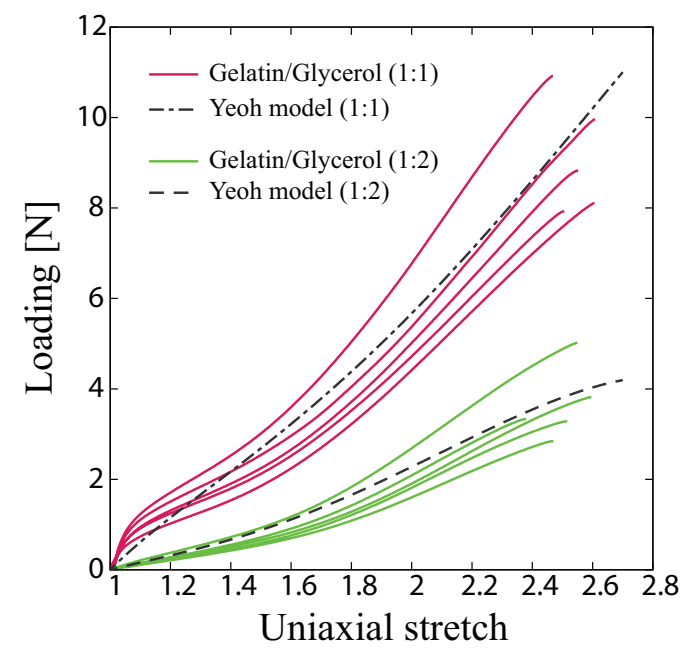

Fig. 3. Characterization results for the gelatin/glycerol material samples. (a) Variation in the mass of the compositions over time. The water content takes equilibrium after $48 \mathrm{~h}$. (b) Loading as a function of uniaxial stretch for the two different compositions. Dashed lines represent calculated result using Eq. 8.

where $\mu$ is the shear modulus. We approximate the Poisson's ratio of the gelatin-glycerol material $\nu$ as 0.49 , which is the case of natural rubbers [18]. The Young's modulus of the material $E$ is then given as

$$
E=2 \mu(1+\nu) .
$$

\section{Results and discussion}

Fig. 3(b) plots the result of the tensile testing for two different gelatin(GEL)/glycerol(GLY) composition of 1:1 and 1:2. The dashed lines shown in the figure are the calculated loading based on Eq. 8. The material constants and properties obtained are summarized in Table I and Table II, respectively. The measured Young's modulus is $2.7 \pm 0.5 \mathrm{MPa}$ for GEL/GLY $=1: 1$, and $0.7 \pm 0.2 \mathrm{MPa}$ for GEL/GLY $=1: 2$, respectively. These values of the modulus are in the same range of silicone elastomers used for soft robots (0.125 MPa for Smooth-On Ecoflex 00-30 [19], and 1.3-3.0 MPa for Dow Corning Sylgard 184 [20]). Also, it can be seen that the composition ratio changes the modulus; larger the glycerol contents, softer the material is. Therefore, desired modulus may be achieved by adjusting the ratio of gelatin and glycerol.

Similar to the Young's modulus, the tensile strength for the two compositions $(9.3 \pm 1.2 \mathrm{MPa}$ for GEL/GLY $=1: 1$, and $3.7 \pm 0.9 \mathrm{MPa}$ for GEL/GLY = 1:2) are in the same range of silicone elastomers (3.5-7.7 MPa for Sylgard 184 [20], and 1.4 MPa for Ecoflex 00-30 [21]).

The elongation at break obtained for the two is almost the same value; $154.8 \pm 6.2 \%$ for GEL/GLY $=1: 1$, and $150.3 \pm 8.2 \%$ for GEL/GLY $=1: 2$, respectively. The value is reasonable for GEL/GLY $=1: 1$ because silicone elastomers with high modulus exhibit similar elongation at break (80$170 \%$ for Sylgard 184 [20]). On the other hand, the value of GEL/GLY $=1: 2,150.3 \pm 8.2 \%$ is significantly smaller than those of soft elastomers (900\% for Ecoflex 00-30 [21]). This
TABLE I

MATERIAL CONSTANTS OF GELATIN(GEL)-GLYCEROL(GLY) MATERIAL

\begin{tabular}{ccc}
\multicolumn{3}{c}{ OBTAINED FROM TENSILE TESTING } \\
Constant $[\mathrm{MPa}]$ & GEL/GLY $(1: 1)$ & GEL/GLY $(1: 2)$ \\
\hline$C_{1}$ & $0.45 \pm 0.09$ & $0.12 \pm 0.03$ \\
$C_{2}$ & $5.72 \pm 1.08 \times 10^{-2}$ & $4.60 \pm 0.87 \times 10^{-2}$ \\
$C_{3}$ & $-0.21 \pm 0.11 \times 10^{-2}$ & $-0.33 \pm 0.07 \times 10^{-2}$ \\
\hline
\end{tabular}

TABLE II

MATERIAL PROPERTIES OF GELATIN(GEL)-GLYCEROL(GLY) MATERIAL OBTAINED FROM TENSILE TESTING

\begin{tabular}{ccc} 
Property & GEL/GLY $(1: 1)$ & GEL/GLY (1:2) \\
\hline Young's modulus [MPa] & $2.7 \pm 0.5$ & $0.7 \pm 0.2$ \\
Tensile strength [MPa] & $9.3 \pm 1.2$ & $3.7 \pm 0.9$ \\
Elongation at break [\%] & $154.8 \pm 6.2$ & $150.3 \pm 8.2$ \\
\hline
\end{tabular}

may have resulted from the rough edge of the samples caused by heating during the laser cutting. The rough-edged sample would have tearing before it arrives the true elongation at break of the material. A potential solution for this issue is to prepare the samples using only molding process.

\section{DEVELOPMENT AND CHARACTERIZATION OF SOFT PNEUMATIC GELATIN ACTUATOR}

Based on the results obtained in the previous section, we developed a proof-of-concept fully edible soft pneumatic actuator using the gelatin-glycerol material.

\section{A. Design and fabrication}

The actuator shown in Fig. 1 has multiple, half-separated chambers in the monolithic structure, similar to the configuration initially reported in [22]. As shown in Fig. 4(a), when pressurized air is injected, the inflation of the chambers happens in the upper part (shifted position from the neutral plane), resulting in a bending of the structure. The dimensions of the device were $90 \mathrm{~mm}$ in length, $20 \mathrm{~mm}$ in 

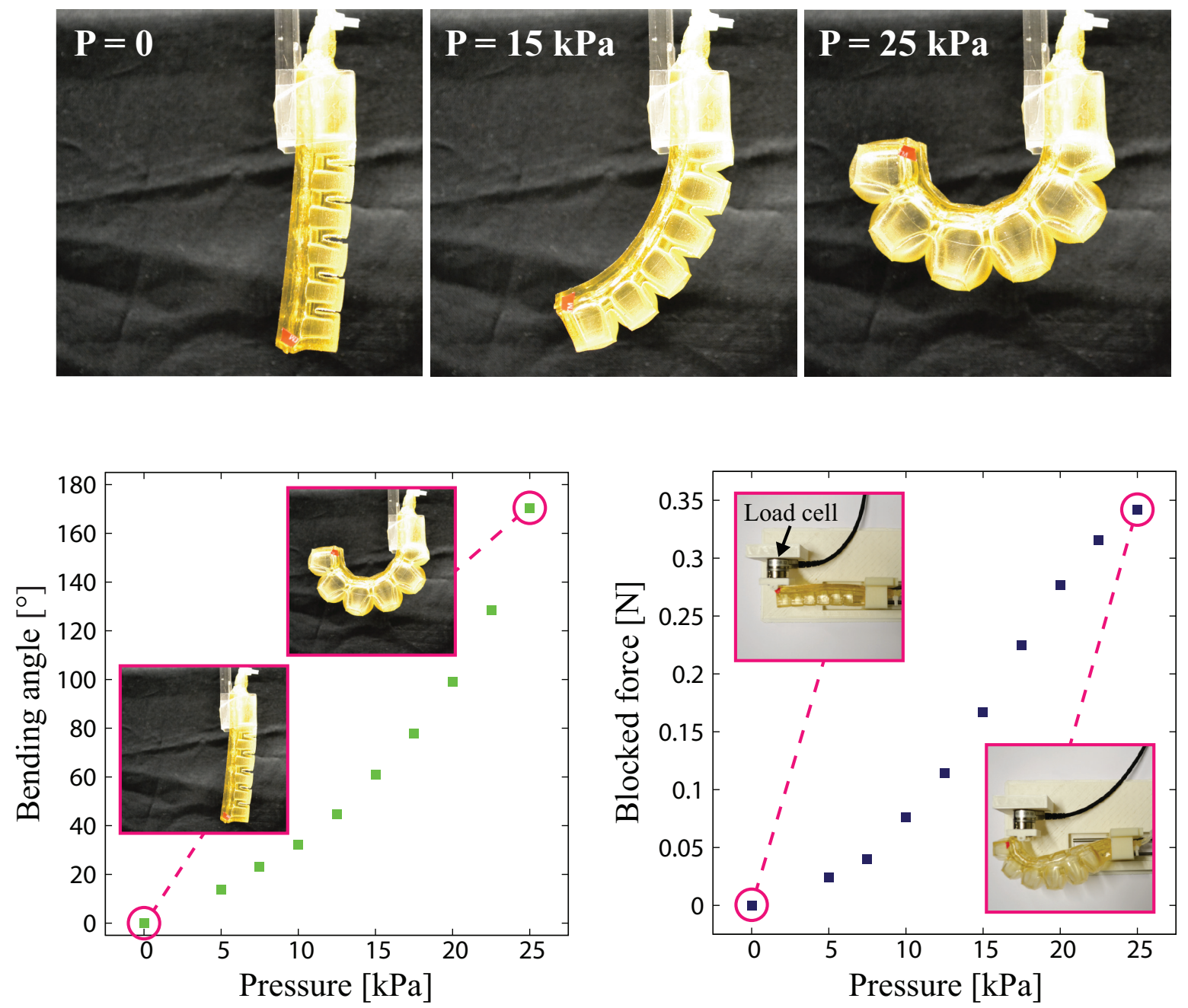

Fig. 4. Characterization results of the soft pneumatic gelatin actuator. (a) Bending of the actuator in different pressured states. P stands for the gauge pressure. (b) Bending angle as a function of the applied pressure. A bending angle of $170.3^{\circ}$ was observed at $25 \mathrm{kPa}$. (b) Blocked force as a function of the applied pressure. A force of $0.34 \mathrm{~N}$ was measured at $25 \mathrm{kPa}$.

width, and $17 \mathrm{~mm}$ in thickness. The actuator was fabricated based on a molding method commonly used for silicone elastomer based pneumatic actuators. We used the composition of gelatin/glycerol/water $=1: 2: 3$, followed by the same preparation method explained in Section II.

\section{B. Bending angle and blocked force}

The bending angle of the actuator was defined as the tip angle difference from the initial angle, which was recorded by a CMOS camera. The blocked force of the actuator was measured by putting a load cell (Nano 17, ATI Industrial Automation) on the tip in the way blocking the bending actuation (Fig. 4(c) inset) In these characterizations, pressurized air of up to $25 \mathrm{kPa}$ was applied via a setup consisted of a valve, a compressor, and a computer running LabView.

\section{Results and discussion}

Fig. 4(b) plots the bending angle as a function of the applied pressure. The angle increased with the pressure, and took a value $170.3^{\circ}$ at $25 \mathrm{kPa}$. Similar trend was observed
TABLE III

COMPARISON OF BLOCKED FORCE FOR THE EDIBLE ACTUATOR AND OTHER ACTUATORS

\begin{tabular}{cc} 
Pneumatic actuator & Blocked force [N] \\
\hline Our edible actuator & 0.3 at $25 \mathrm{kPa}$ \\
Elastomer based actuator in [23] & 0.2 at $25 \mathrm{kPa}$ \\
Elastomer based actuator in [24] & 0.4 at $25 \mathrm{kPa}$ \\
Elastomer based actuator in [25] & 0.5 at $40 \mathrm{kPa}$ \\
Elastomer based actuator in [26] & 0.5 at $50 \mathrm{kPa}$ \\
\hline
\end{tabular}

in the blocked force, and a force value of $0.34 \mathrm{~N}$ was recorded at $25 \mathrm{kPa}$. These results represent the fact that the performance of the actuator, the actuation angle and the force, are pressure-controllable. We found our edible soft actuator exhibited comparable performance to existing silicone elastomer based actuators, as summarized in Table III. During the test, we observed the actuator showed fast motion $(\sim 0.5 \mathrm{~s})$, and was capable of withstand numerous cycles of actuation (see supplemental video). 
a
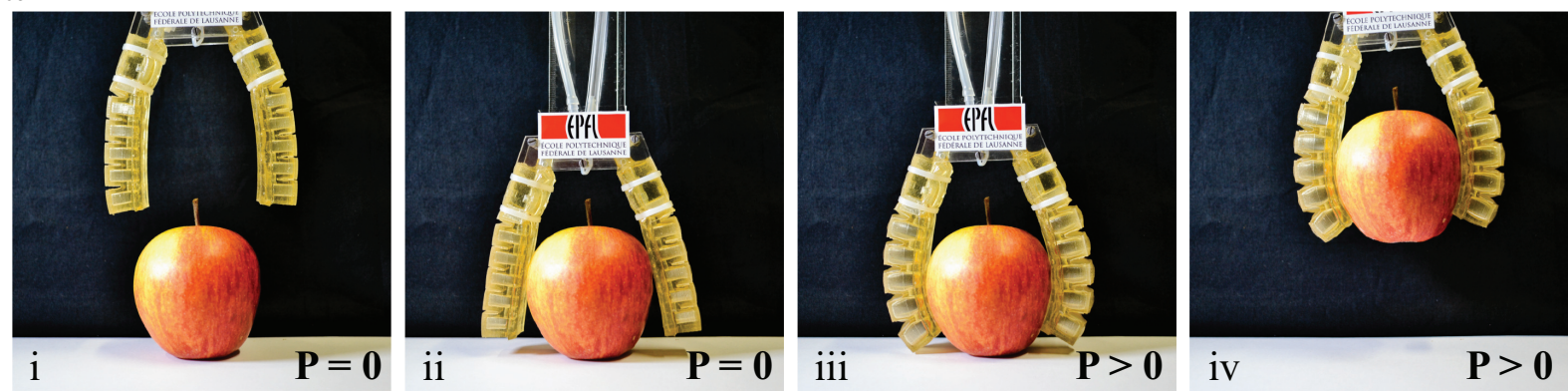

b

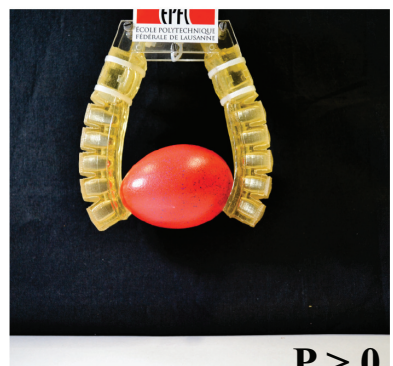

c

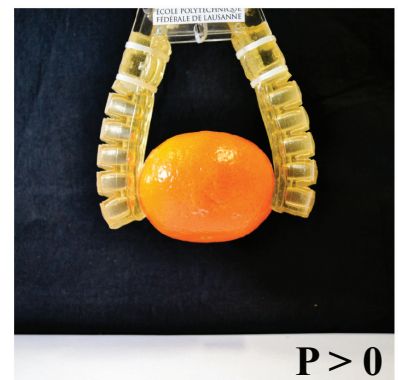

d

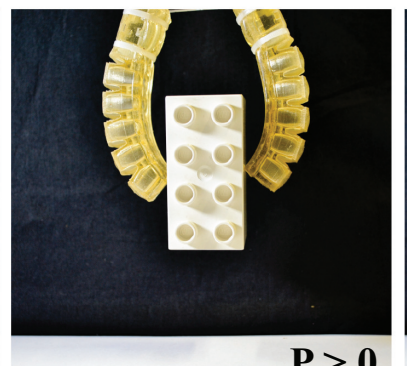

e

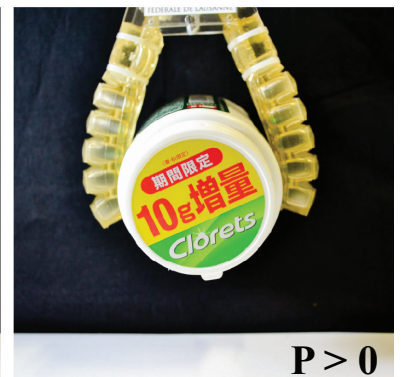

Fig. 5. Gripper grasping test. P stands for the gauge pressure. (a-i) The gripper is placed above the object, here an apple mass of 95.6 g. (a-ii) The device approaches the object. (a-iii) Applying pressure conforms the adaptation of the fingers. (a-iv) The actuated force of the gripper makes it possible to picked up the object. The gripper demonstrated handling of other objects: (b) a boiled egg (47.7 g), (c) an orange (104.8 g), (e) A LEGO brick (25.7 g), and (f) A bottle of chewing gums (153.1 g).

\section{DEMONSTRATION OF ROBOTIC APPLICATION}

In order to demonstrate the applicability of the edible soft pneumatic actuator to robotic application, we integrated the two actuators into a form of two-finger gripper and performed grasping test for various objects. The gripper, shown in Fig. 5(a-i-ii), has the open-finger at zero pressure applied. When pressurized air is injected $(\sim 20 \mathrm{kPa}$ in this demonstration), the fingers adapt to the object, here an apple $(95.6 \mathrm{~g})$, and provide enough force to lift it up (Fig.5(a-iiiiv)). As shown in Fig. 3(b-f), we observed the gripper was able to grasp and lift up varied objects: a boiled egg (47.7 $\mathrm{g}$ ), an orange (104.8 g), a LEGO brick (25.7 g), and a bottle of chewing gums $(153.1 \mathrm{~g})$. This versatility of the gripper illustrates high performance of our edible actuator.

\section{Discussion}

The edible material used for the actuator, the gelatinglycerol material, shows similar mechanical properties to those of silicone elastomers. Assessment of other characteristics such as durability and viscoelasticity with varied composition ratios will provide more insight about applicability of the material for robotic applications. Environmental conditions such as humidity and temperature, are also important aspects of robots that operate in uncontrolled environments. As discussed in Section II, the gelatin-glycerol material appears to reach an equilibrium water content (Fig. 3(a)), suggesting that the mechanical properties may change corresponding to humidity of the surrounding air. However, this is not a major problem because edible coatings to preserve the water content are already available [27]. Temperature may also effect to the mechanical properties because the melting temperature of gelatin is around $35^{\circ} \mathrm{C}$. The melting point can be higher once plasticizers and other proteins are added. For example, a material of gelatin, chitosan (a polysaccharide), and glycerol has a melting point of $67^{\circ} \mathrm{C}$ [28]. Investigation of above mentioned characteristics will contribute towards the improved design of the actuators and robots, and their applicable tasks.

The soft pneumatic edible actuator described here displays performance comparable to actuators made of silicone elastomer based even if the actuator design described here was not yet optimized. Higher performance may be expected if the actuator is designed with aid of analytical modeling [29] or FEM [25] where the material properties obtained from the characterization are incorporated. This will also enable the design of actuators of different geometries and sizes. For the future generation of the edible actuators, further characterization on cycles (i.e., durability), repeatability, hysteresis, effect of temperature and humidity will be performed. Given the melting feature of gelatin, our actuator could be capable of self-healing and become re-usable, which are added capabilities that existing soft pneumatic actuators usually do not exhibit. The edible robotic gripper described here shows possibility of creating edible robots based on these materials. Given the simplicity of the actuator design, it could be implemented to many different types of 
robots. Along with all the functionalities-biodegradability, biocompatibility, environmental sustainability, digestibility, metabolizability, selfheal ability, and re-usability-those edible robots could bring novel applications.

For example, as discussed in literature [30] about the animal navigation in wild, fully edible robots would help to study how wild animals collectively behave. The robots could also take a role of animals prey to observe their hunting behaviors, or to train protected animals to do predation. Once medical components are mixed into the edible composition, the robots could help preservation of wild animals or heal inside of the human body. When edible robots can be metabolized, they also function as energy storage providing an advantage in terms of increased payload with respect to non-edible robots that must be loaded with a food payload. This would be effective in rescue scenarios where the metabolizable robots can reach survivors in isolated places like inside a crevice or up on mountain. Last, but not least, since edible materials can generate electric energy [6]-[9], one could envisage autophagy (self-eating) function, like that of octopus [31], to extend their lifetime.

\section{CONCLUSION}

In this paper, we proposed and characterized a gelatinglycerol material as a candidate for soft edible actuator material. The characterization results showed that the material had mechanical properties that were mostly in the same range as those of the conventional silicone elastomers. Based on the results, we also developed a proof of concept, fully edible, soft gelatin pneumatic actuator. The edible actuator exhibited comparable performance to silicone elastomer based actuators. The two actuators were then integrated to form a two-finger gripper. With this gripper, we demonstrated handling of various objects. We believe that edible actuators, combined with other edible electronics and sensors, could pave the way towards the new field of edible robotics.

\section{ACKNOWLEDGMENTS}

This work was supported by the Swiss National Centre of Competence in Research (NCCR) Robotics.

\section{REFERENCES}

[1] D. Rus and M. T. Tolley, "Design, fabrication and control of soft robots," Nature, vol. 521, no. 7553, pp. 467-475, 2015.

[2] D. Trivedi, C. D. Rahn , W. M. Kier and I. D. Walker, "Soft robotics: biological inspiration, state of the art, and future research," Applied Bionics and Biomechanics, vol. 5, no. 3, pp. 99-117, 2008.

[3] M. Irimia-Vladu, N. S. Sariciftci and S. Bauer, "Exotic materials for bio-organic electronics,", Journal of Materials Chemistry, vol. 21, no. 5, pp. 1350-1361, 2011.

[4] M. Irimia-Vladu et al., "Biocompatible and biodegradable materials for organic field-effect transistors," Advanced Functional Materials, vol. 20, no. 23, pp. 40694076, 2010.

[5] H. Tao et al., "Silk-based conformal, adhesive, edible food sensors," Advanced Materials, vol. 24, no. 8, pp. 1067-1072, 2012.

[6] Y. J. Kim, S.-E. Chun, J. Whitacre and C. J. Bettinger, "Selfdeployable current sources fabricated from edible materials," Journal of Materials Chemistry B, vol. 1, no. 31, pp. 3781-3788, 2013.

[7] Z. Li et al., "Towards high power high energy aqueous sodium-ion batteries: the NaTi 2(PO4)3/Na0.44MnO2 system," Advanced Energy Materials, vol. 3, no. 3, pp. 290-294, 2013.
[8] Y. J. Kim et al., Biologically derived melanin electrodes in aqueous sodium-ion energy storage devices, Proceedings of the National Academy of Sciences, vol. 100, no. 51, pp. 2091220917, 2013.

[9] W. Wu, A. Mohamed, and J. F. Whitacre, "Microwave synthesized NaTi2(PO4)3 as an aqueous sodium-ion negative electrode," Journal of The Electrochemical Society, vol. 160, no. 3, pp. 497-504, 2013.

[10] A. Keller, L. Stevens, G. G. Wallace, and M. in het Panhuis, "3D printed edible hydrogel electrodes," Biomaterials and Soft Materials, vol. 1 , no. 8, pp. 527-532, 2015.

[11] W. Xu et al., "Foodmaterialsbased edible supercapacitors,", Advanced Materials Technologies,vol. 1, no. 3, p. 1600059, 2016.

[12] L. D. Chamber, J. Winfield, I. Ieropoulos, and J. Rossiter, 'Biodegradable and edible gelatine actuators for use as artificial muscles," in Proc. SPIE Electroactive Polymer Actuators and Devices, San Diego, USA, March 2014.

[13] J. Rossiter, J. Winfield, and I. Ieropoulos "Here today, gone tomorrow: biodegradable soft robots," in Proc. SPIE Electroactive Polymer Actuators and Devices, Las Vegas, USA, March 2016.

[14] S. Miyashita et al., "Ingestible, controllable, and degradable origami robot for patching stomach wounds," in IEEE International Conference on Robotics and Automation, Stockholm, Sweden, May 2015.

[15] P. Bergo, P. J. A. Sobral, "Effects of plasticizer on physical properties of pigskin gelatin films," Food Hydrocolloids, vol. 21, no. 8, pp. 12851289, 2007.

[16] ASTM D412-16, "Standard test methods for vulcanized rubber and thermoplastic elastomers-tension," ASTM International, West Conshohocken, PA, 2016.

[17] O. H. Yeoh, "Some forms of the strain energy function for rubber," Rubber Chemistry and technology, vol. 66, no. 5, pp. 754-771, 1993.

[18] I. M. Ward and J. Sweeney, Mechanical properties of solid polymers, John Wiley \& Sons, 2012.

[19] Y.-L. Park et al., "Hyperelastic pressure sensing with a liquidembedded elastomer," Journal of Micromechanics and Microengineering, vol. 20, no. 12, p. 125029, 2010.

[20] I. D. Johnston, D. K. McCluskey, C. K. L. Tan, and M. C. Tracey, "Mechanical characterization of bulk Sylgard 184 for microfluidics and microengineering," Journal of Micromechanics and Microengineering, vol. 24, no. 3, p. 035017, 2014.

[21] Smooth-On, Inc., "Ecoflex 00-30 Product Information," Internet: https://www.smooth-on.com/products/ecoflex-00-30/, [February 26, 2017].

[22] K. Ogura, S. Wakimoto, K. Suzumori, and Y. Nishioka, "Micro pneumatic curling actuator - nematode actuator -," in IEEE International Conference on Robotics and Biomimetics, Bangkok, Thailand, February 2009.

[23] B. Mosadegh et al., "Pneumatic networks for soft robotics that actuate rapidly," Advanced Functional Materials, vol. 24, no. 15, pp. 21632170, 2014.

[24] P. Polygerinos et al., "Towards a soft pneumatic glove for hand rehabilitation," in IEEE/RSJ International Conference on Intelligent Robots and Systems, Tokyo, Japan, November 2013.

[25] P. Moseley et al., "Modeling, design, and development of soft pneumatic actuators with finite element method," Advanced Engineering Materials, vol. 18, no. 6, pp. 978-988, 2016.

[26] K. C. Galloway, P. Polygerinos, C. J. Walsh, and R. J. Wood, "Mechanically programmable bend radius for fiber-reinforced soft actuators," in 16th International Conference on Advanced Robotics, Montevideo, Uruguay, November 2013.

[27] B. Marelli, M. A. Brenckle, D. L. Kaplan, and F. G. Omenetto, "Silk fibroin as edible coating for perishable food preservation," Scientific Reports, vol. 6, p. 25263, 2016.

[28] I. S. Arvanitoyannis, A. Nakayama, and S. Aiba, "Chitosan and gelatin based edible films: state diagrams, mechanical and permeation properties," Carbohydrate Polymers, vol. 34, no. 4, pp. 371-382, 1998.

[29] V. Cacucciolo et al., "Modelling the nonlinear response of fibrereinforced bending fluidic actuators," Smart Materials and Structures, vol. 25 , no. 10, p. 105020, 2016.

[30] J. Krause, A. F. T. Winfield, and J.-L. Deneubourg, "Interactive robots in experimental biology," Trends in ecology \& evolution, vol. 26, no. 7, pp. 369-375, 2011.

[31] B. U. Budelmann, "Autophagy in octopus," South African Journal of Marine Science, vol. 20, no. 1, pp. 101-108, 1998. 